\title{
The determination of the critical value of the likelihood ratio test
}

\author{
Hongliang Kang
}

\begin{abstract}
This paper studies the likelihood ratio test critical values determined by the three methods. The first one is to build the likelihood than a function of the relationship between statistics and distribution of the known statistics, by a critical value of the known statistics, according to the function relation, indirectly calculated likelihood ratio statistic critical value; secondly, using Monte Carlo simulation; third, when the sample size $n$ is large, the likelihood ratio statistics limit distribution approximation is given. Based on the three kinds of methods to solve the likelihood ratio test statistic distribution is unknown and in practical computation is difficult to determine the critical value.
\end{abstract}

Index Terms - likelihood ratio test,critical value, Monte Carlo simulation,distribution, likelihood ratio statistic.

MSC(2010):-39A10, 39A12

\section{INTRODUCTION}

Likelihood ratio test is a widely used test method. However, there is no uniform and accurate distribution of likelihood ratio test statistics so far in general, so it is very important to determine the critical value of likelihood ratio test.

\section{PRELIMINARY}

Define 2.1 maximum likelihood estimator and assume that the probability density of the population is $p(x ; \theta) \theta \in \Theta, \theta$ a parameter vector consisting of an unknown parameter or several unknown parameters, $\Theta$ a parameter space, $x_{1}, \ldots, x_{n}$ a sample from the population, and regard the joint density function of the sample as a $\theta$ function.

$L(\theta)$ is called the likelihood function of a sample. If a statistic satisfies

$$
, L(\hat{\theta})=\max _{\theta \in \Theta} L(\theta)
$$

The maximum likelihood estimator is called MLE(maximum likelihood estimate)..

Define 2.2 Generalized likelihood ratio is defined as a sample from the density function. The following test questions are considered.

$$
H_{0}: \theta \in \Theta_{0} \text { vs } H_{1}: \theta \in \Theta_{1}=\Theta-\Theta_{0} .
$$
order

$$
\Lambda\left(x_{1}, \ldots, x_{n}\right)=\frac{\sup _{\theta \in \Theta} p\left(x_{1}, \ldots, x_{n} ; \theta\right)}{\sup _{\theta \in \Theta_{0}} p\left(x_{1}, \ldots, x_{n} ; \theta\right)},
$$

Then we call $\Lambda\left(x_{1}, \ldots, x_{n}\right)$ the statistic the likelihood ratio

Hongliang Kang, Department of Mathematics, Northwest Normal University, Lanzhou, Gansu, China, Mobile No18419067896 hypothesized above, sometimes called the generalized likelihood ratio.

Define 2.3 Likelihood ratio test should use the above likelihood ratio statistics $\Lambda\left(x_{1}, \ldots, x_{n}\right)$ as test statistics, and take its rejection domain as $W=\left\{\Lambda\left(x_{1}, \ldots, x_{n}\right) \geq c\right\}$, where the critical value c satisfies

$P_{\theta}\left(\Lambda\left(x_{1}, \ldots, x_{n}\right) \geq c\right) \leq \alpha, \quad \forall \theta \in \Theta_{0}$,

This test is called likelihood ratio test (LRT.) with a significant level $\alpha$.

Define 2.4 $\chi^{2}$ Distribution (chi-square distribution) assumes $X_{1}, X_{2}, \ldots, X_{n}$ an independent and identical distribution in the standard normal distribution $N(0,1)$, then the distribution $\chi^{2}=X_{1}^{2}+\cdots+X_{n}^{2}$ is called the distribution $\chi^{2}$ of degree of freedom $n$, denoted as $\chi^{2} \sim \chi^{2}(n)$.

Define 2.5 Griffin's theorem assumes that $x_{1}, x_{2}, \cdots, x_{n}$ a sample is taken from the population distribution function $F(x)$ and $F_{n}(x)$ is its empirical distribution function. At that time $n \rightarrow \infty$, there are

$P\left(\sup _{-\infty<x<\infty}\left|F_{n}(x)-F(x)\right| \rightarrow 0\right)=1$.

\section{CALCULATE THE CRITICAL VALUE INDIRECTLY BY USING FUNCTION RELATION.}

Let's take the normal population as an example to illustrate this method briefly.

Example 3.1.1, normal population, known variance, test problem

$H_{0}: \mu=\mu_{0}, \quad H_{1}: \mu=\mu_{1}\left(\mu_{1}>\mu_{0}\right)$.

Likelihood ratio $\Lambda\left(x_{1}, \ldots, x_{n}\right)=\frac{p\left(x_{1}, \ldots, x_{n} ; \mu_{1}\right)}{p\left(x_{1}, \ldots, x_{n} ; \mu_{0}\right)}$

$=\frac{\left(\frac{1}{\sqrt{2 \pi} \sigma}\right)^{n} \exp \left\{-\frac{1}{2 \sigma^{2}} \sum_{i=1}^{n}\left(x_{i}-\mu_{1}\right)^{2}\right\}}{\left(\frac{1}{\sqrt{2 \pi} \sigma}\right)^{n} \exp \left\{-\frac{1}{2 \sigma^{2}} \sum_{i=1}^{n}\left(x_{i}-\mu_{0}\right)^{2}\right\}}$

$=\exp \left\{-\frac{1}{2 \sigma^{2}} \sum_{i=1}^{n}\left[\left(x_{i}-\mu_{1}\right)^{2}-\left(x_{i}-\mu_{0}\right)^{2}\right]\right\}$ 
$\exp \left\{\frac{\mu_{1}-\mu_{0}}{2 \sigma^{2}} \sum_{i=1}^{n}\left(2 x_{i}-\mu_{1}-\mu_{0}\right)\right\}$
$=\exp \left\{\frac{\sqrt{n}\left(\mu_{1}-\mu_{0}\right)}{\sigma} \frac{\bar{x}-\mu_{0}}{\sigma / \sqrt{n}}-\frac{n\left(\mu_{1}-\mu_{0}\right)^{2}}{2 \sigma^{2}}\right\}$.

Order $U=\frac{\bar{X}-\mu_{0}}{\sigma / \sqrt{n}}$,
$\Lambda\left(x_{1}, \ldots, x_{n}\right)=\exp \left\{\frac{\sqrt{n}\left(\mu_{1}-\mu_{0}\right)}{\sigma} U-\frac{n\left(\mu_{1}-\mu_{0}\right)^{2}}{2 \sigma^{2}}\right\}$

Because it is known $\mu_{0}, \mu_{1}, \sigma^{2}$ and $\mu_{1}>\mu_{0}$, so $\Lambda\left(x_{1}, \ldots, x_{n}\right)$ is a monotone increasing function $U$. when the test statistic $c_{1}=\mu_{1-\alpha}$ is taken, the critical value $U=\frac{\bar{X}-\mu_{0}}{\sigma / \sqrt{n}}$ and $c_{1}=\mu_{1-\alpha}$ the rejection field $W=\left\{U: U>\mu_{1-\alpha}\right\}$ can be obtained.

Because of $\Lambda\left(x_{1}, \ldots, x_{n}\right)$ the monotonous increasing function, we can see that the critical value of likelihood ratio test is

$c=\exp \left\{\frac{\sqrt{n}\left(\mu_{1}-\mu_{0}\right)}{\sigma} \mu_{1-\alpha}-\frac{n\left(\mu_{1}-\mu_{0}\right)^{2}}{2 \sigma^{2}}\right\}$

The domain of rejection is

$W=\left\{\Lambda\left(x_{1}, \ldots, x_{n}\right): \Lambda\left(x_{1}, \ldots, x_{n}\right)>\exp \left\{\frac{\sqrt{n}\left(\mu_{1}-\mu_{0}\right)}{\sigma} \mu_{1-\alpha}-\frac{n\left(\mu_{1}-\mu_{0}\right)^{2}}{2 \sigma^{2}}\right\}\right\}$.

Example 3.1.2 for normal population, unknown variance, test problem

$H_{0}: \mu=\mu_{0}, \quad H_{1}: \mu \neq \mu_{0}$.

likelihood ratio $\Lambda\left(x_{1}, \ldots, x_{n}\right)=\frac{\sup _{\theta \in \Theta} p\left(x_{1}, \ldots, x_{n} ; \theta\right)}{\sup _{\theta \in \Theta_{0}} p\left(x_{1}, \ldots, x_{n} ; \theta\right)}$,

Here,

$\Theta=\left\{\left(\mu, \sigma^{2}\right),-\infty<\mu<\infty, \sigma^{2}>0\right\}$,

$\Theta_{0}=\left\{\left(\mu_{0}, \sigma^{2}\right), \sigma^{2}>0\right\}$.

When $\mu, \sigma^{2}$ unknown, ,the maximum likelihood estimators are respectively $\hat{\mu}=\bar{x}, \quad \hat{\sigma}^{2}=\frac{1}{n} \sum_{i=1}^{n}\left(x_{i}-\bar{x}\right)^{2}$.

When known $\mu=\mu_{0}$, the maximum likelihood estimate $\sigma^{2}$ is

$\hat{\sigma}_{0}^{2}=\frac{1}{n} \sum_{i=1}^{n}\left(x_{i}-\mu_{0}\right)^{2}$.
$\Lambda\left(x_{1}, \ldots, x_{n}\right)$

$\frac{\left(\frac{1}{\sqrt{2 \pi} \hat{\sigma}}\right)^{n} \exp \left\{-\frac{1}{2 \hat{\sigma}^{2}} \sum_{i=1}^{n}\left(x_{i}-\bar{x}\right)^{2}\right\}}{\left(\frac{1}{\sqrt{2 \pi} \hat{\sigma}_{0}}\right)^{n} \exp \left\{-\frac{1}{2 \hat{\sigma}_{0}^{2}} \sum_{i=1}^{n}\left(x_{i}-\mu_{0}\right)^{2}\right\}}$

$=\left(\frac{\hat{\sigma}_{0}^{2}}{\hat{\sigma}^{2}}\right)^{\frac{n}{2}}=\left(1+\frac{n\left(\bar{x}-\mu_{0}\right)^{2}}{(n-1) S^{2}}\right)^{\frac{n}{2}}$.

So the likelihood ratio $T=\frac{\bar{x}-\mu_{0}}{S / \sqrt{n}}$ is

$\Lambda\left(x_{1}, \ldots, x_{n}\right)=\left(1+\frac{T^{2}}{n-1}\right)^{\frac{n}{2}}$.

\section{THE SUMMARY OF METHODS}

The method of calculating the critical value by using the function relation is more accurate, and the amount of calculation is less, which is generally desired by us. But it has limitations. Only when the likelihood ratio statistic establishes the function relation with a certain statistic of the known distribution, and the function is monotonous, can this method be used. Obviously, its applicable scope is very clear. There are no two broad encores.

Monte Carlo simulation method for calculating the critical value is applicable to a wide range of applications, and it is more convenient to operate with a computer, but the amount of calculation is large, the calculated results are only approximate values, and the results will also be related to the model differences selected by individuals.

The approximate method for calculating the critical value of asymptotic distribution has a wide scope of application, and the amount of calculation is small, so it is easy to grasp. But the critical value determined by the method is not very accurate, so it can only be said that the test is roughly carried out, and the reliability is relatively low.

\section{CONCLUDING REMARKS}

We use the above two methods to determine the critical value of likelihood ratio test for a specific problem.

Likelihood ratio test is a widely used test method, because its test statistic distribution is uncertain, it brings some troubles to the hypothesis test problem. To solve the problem of hypothesis test, the key is to determine the critical value, through which to judge whether to reject the original hypothesis. Especially important.

In this paper, three methods to determine the critical value are given, and the critical value problem of likelihood ratio test is discussed briefly. The method of calculating the critical value by using the function relation is discussed in detail Moreover, the critical value calculated by this method is accurate, and the concrete determination process is simple and understandable. Readers can understand this square emphatically. The latter two methods are discussed briefly. The Monte Carlo simulation algorithm is a practical method 
which can simulate and calculate the parameters of various unknown distributions. However, this method requires a high level of computer. We can understand its general idea first in this paper for the future. The method of calculating the critical value by using asymptotic distribution is easy to understand, but it requires a large sample size, and the critical value is not precise. When we encounter problems which can not be solved by the above two methods, we can roughly estimate the critical value by this method.

This paper only summarizes three methods of determining the critical value of likelihood ratio test. Because of my limited level, the Monte Carlo simulation method only tells its basic idea. The program and operation of computer simulation are not given. I believe that with the improvement of our knowledge level, we will learn to operate this method in detail. In order to better understand the problem of determining the critical value of likelihood ratio test and perfect the statistical inference module of likelihood ratio test, this paper only briefly introduces three methods and other methods have not been explained.

\section{REFERENCES}

[1]Cheng Ping. Several remarks on maximum likelihood estimation and likelihood ratio test $[\mathrm{J}]$. application probability statistics, 2003,01:55-59.

[2] Wang Yan. Estimation of multiple normal population parameters and likelihood ratio test [D]. Liaoning University of Technology, 2012.

[3] Tang Niansheng. Likelihood ratio test for multiple outliers in uniform samples [J].Journal of Yunnan University (Natural Science Edition), 1998, S2:218-222.

[4] Song Lixin, Zhao Li. Two general equivalent generalized likelihood ratio tests [J]. university mathematics, 2005,02:91-94.

[5] Wang Yan, Li Shuyou, Mi Ying. Likelihood Ratio Test for Multiple Normal Population Parameters [J].Journal of Liaoning University of Technology (Natural Science Edition), 2011,05:336-340.

[6] Liu Binghui. Compressibility of likelihood ratio test and decomposition of test statistics [D].Northeast Normal University, 2006.

[7] Wang Rui generalized likelihood ratio test in two normal populations [J] science and technology horizon, 2012,32:39-49.

Hongliang Kang, Collage of Mathematics and Statistics, Northwest Normal University, Lanzhou, China, Mobile 86- 18419067896. 